diagnosis are presented for osteogenesis imperfecta, a model disease for which various different clinical and biochemical forms arise from different mutations in the type I procollagen gene.

Chapters 9 to 11 provide a very useful synopsis on the important broader issues of education, counselling, ethical principles, and the consumer's perspective of prenatal diagnosis, which would be of interest to all professionals involved in prenatal diagnosis. There is an appendix which summarises the principles and practices of neonatal screening; details are given for specific disorders. The glossary contains helpful definitions of medical and technical terms and phrases.

In summary, the Handbook is well structured and may be useful to a range of health care professionals, but because of the broad focus it lacks detail and should not be regarded as a comprehensive text. It is straightforward to read and understand and may be of particular value to trainees in clinical genetics and to genetic nurse specialists. A very positive aspect is the emphasis given to the importance of ethical issues, genetic counselling, and the consumer's viewpoint in the field of prenatal diagnosis.

SARAH SLANEY

Atlas of Whole Chromosome Paint Probes. Normal Patterns and Utility for Abnormal Cases. S M Jalal, M E Law, G W Dewald. (Pp 145; \$49.50.) Rochester: Mayo Foundation for Medical Education and Research. 1995. ISBN 0-9627865-4-3.

The authors defend the need for this Atlas on the grounds that the use of whole chromosome paints has become almost routine for the elucidation of some cases in a routine cytogenetics laboratory and that there is no reference source of normal patterns. To this end, using paints for all of the chromosomes, they have produced a colourful and informative review which provides a ready reference for the new initiate. However, there are limitations to the Atlas. All of the WCPs (sic) are from a single source and, at least some, are said to show variation between batches. Paints from alternative sources may not show identical patterns of hybridisation and the method of manufacture may well lead to different distributions of probe intensity. Most cytogeneticists would, I am sure, evaluate a new WCP on control material before its use on abnormal material so would avoid potential pitfalls, but this also detracts from the usefulness of the Atlas for this purpose.

The section of the Atlas concerning "Representative Abnormal Cases" provides a good review of the various problems that can be resolved by the use of WCPs. Sometimes new technologies lead to more complicated ways of dealing with old problems, and that is certainly the case for some of the cases used as examples. However, the approach cannot be faulted and the photographs are certainly eye catching.

This is more of a "coffee table" book to impress those still unsure of.the merits of FISH technology than a reference book for those who use the technology on a day to day basis.

TONY ANDREWS

\section{NEW SOFTWARE}

Cyrillic 2.0 for Pedigree Drawing. Cyril Chapman. ( $£ 399.00$. Special 5 pack, 10 pack, and department licence prices.) Oxford: Cherwell Scientific Publishing. 1995. Serial No 050-200-1012.

I first encountered the Cyrillic program when its author, Cyril Chapman, spent some time in Cambridge in 1989; it was running on an old Amstrad 1640 under the GEM operating system but even then was obviously a useful tool. So it was with pleasure that I accepted the offer of reviewing the most current release of Cyril's program. The program has come a long way from those early days, but has managed to retain the feeling of a package designed by a user to do the job that it was intended for on a day to day basis, rather than by a programmer who includes anything that might be useful. As a result it is relatively small for a Windows based program (Cyrillic itself is about $2.3 \mathrm{Mb}$, and complete installation, including example files, takes up less than $4 \mathrm{Mb}$ of hard disk space) and is shipped on a single high density disk. Installation on my computer (a Viglen Genie $486-25 \mathrm{Mz}$, with $4 \mathrm{MB}$ of RAM, running Windows 3.11 ) took very little time and was achieved without problems.

For those who have used Cyrillic 1 this is where the first differences from the new release become apparent; the new version allows the user to establish separate directories for individual projects, each with its own Windows icon and separate data files. This is an improvement that could be of particular value in larger centres to group together families belonging to different research projects or suffering from different diseases. There are several other changes from the first commercial release, and most are improvements that make the program more powerful, yet also simpler and faster.

The program itself remains much as before and can be thought of in two ways; on the one hand it is a functional database that stores information about patients grouped into families, while on the other it is a pedigree drawing program that generates pictures of the relationships among patients. In my opinion, this duality centred on a physical picture of the pedigree is what makes Cyrillic such a useful, and probably unique, program. The program stores each family in a separate file consisting of a picture of the pedigree built up in stages. Initially, a single person is created by clicking the left mouse button anywhere within the computer screen. This generates a symbol at the position of the mouse click; selecting this with the right mouse button then brings up a dialogue box that allows the creation of symbols representing one or more first degree relatives. For example, one could add the patient's partner, parents, sibs, or children to the pedigree. From then on any new members of the pedigree are added by clicking on any of their first degree relatives already on the screen. One vast improvement over earlier versions is that the program now allows the rapid generation of an outline pedigree by automatically creating full sibships, including parents, without each person having to be created separately. Pedigrees are "drawn" on an imaginary "virtual" piece of paper that can be much larger than the visible screen. The visible screen can then be "moved" from one part of the virtual screen to another to create and visualise larger pedigrees than can possibly be viewed at once (the program is said to permit pedigrees containing 10000 people). Personal information, such as birth date, address, DNA storage information, genetic marker results, disease status, etc, can then be entered for any person or group of people by selecting them from the skeleton pedigree at any time after its creation. The families, diseases, and set of genetic markers, which can include biochemical risk factors and VNTR probes, can then be chosen from lists created by the user. These disease and marker data are stored separately from the main program in .DAT files, which can be independently copied, transferred, or grouped together in different directories, or, as in my case, apparently "lost" because I didn't tell the program which directory they were in.

The choice of symbols used to represent people, the lines connecting them, and the data displayed with each symbol are easily customised by the user, and it is always possible to see the full data entered for any person by selecting his/her symbol with the mouse. For example, in families with extensive genetic typing results, any subset can be selected for display below the person's symbol, along with other information such as name and ID number(s). In addition, this new version can automatically work out the haplotypes segregating in the family and assign colour coded bars representing parental chromosomes, visually highlighting any recombinations or other inconsistencies in the data. This haplotyping function is illustrative of Cyrillic's use of uncomplicated tricks to create apparently intricate solutions: the haplotyping algorithm itself is extremely simple and treats each locus as if it were segregating independently. However, because the program can be forced to accept extended parental haplotypes as "true", using the algorithm in conjunction with human intuition allows the user to generate complete chromosomal haplotypes quickly with minimal effort, even in large pedigrees with many markers.

Printing information from the program is also relatively flexible. A direct print of the pedigree on screen is easily obtained using the print option, and this output can contain a wealth of additional information, such as markers used in the family, date of printing, and name of the laboratory, determined by default values entered by the user. However, a more flexible print can be obtained using the Print Preview option (this even gets round the persistently annoying tendency for the program to print a family over two physical pages, even when it would easily fit on one), which allows any pedigree to be drawn on a single page, either as shown on screen or in a circular format, and permits the added information to be moved around to enhance the legibility of the final output. Sub-parts of a pedigree can be displayed and printed separately or even selected and transferred to word processor documents using the Windows Clipboard. In addition to the production of graphical output, Cyrillic can also produce tabulated lists of patient demographics or marker details for a family, arranged alphabetically or by pedigree number. However, the usefulness of this part of the program is severely hampered by the fact 